\title{
Simultaneous temperature and vibration monitoring using an all-PM fiber loop mirror interferometer
}

\author{
D. Leandro*, M. Lopez-Amo \\ Universidad Pública de Navarra, Dept. of Electrical and Electronic Engineering, Campus Arrosadia \\ S/N, E-31006, Pamplona, Spain
}

\begin{abstract}
In this work, an all-polarization maintaining fiber loop mirror interferometer is presented and validated as vibration and temperature sensor without crosstalk between measurands. The system is entirely built using polarization-maintaining (PM) fiber and a PM optical coupler. As a consequence, no polarization controllers are needed in the cavity, significantly simplifying the operation of the system and improving the stability and accuracy of the measurements. The loop comprises three PM fiber sections appropriately fused with an angle offset between them. In this manner, the interference is maximized and the sensing contributions of the two PM fibers used as communications channels are suppressed. The third PM fiber is used as the sensor itself, simultaneously monitoring temperature and mechanical vibration up to 1.2 $\mathrm{kHz}$ by means of an interrogation technique based on fast Fourier transform.
\end{abstract}

Keywords: Fiber loop mirror (FLM), Polarization-maintaining fiber, Sagnac interferometer, photonic crystal fiber (PCF)

\section{INTRODUCTION}

Highly birefringence fiber loop mirrors (HiBi FLMs), also referred as Sagnac interferometers, are a fiber optic configuration successfully employed in many applications such as optical filtering in communications and fiber lasers among others [1]. They have also been used for sensing applications, since the phase of the optical interference is modified by birefringence changes induced, for example, by temperature variations [2]. A direct solution for interrogating interferometers is measuring the wavelength shift of a peak or a valley in the optical spectrum. Nevertheless, this approach cannot be reliably used if several interferometric elements are multiplexed. A valid approach is to use an interrogation technique based on the fast Fourier transform (FFT), which identifies the spatial frequency contributions generated by the different interferometric components present in the interference [3]. This technique, allows the interrogation in a single network of several interferometric contributions with different characteristic spatial frequencies [4-7]. In addition, it also offers a resolution increase of more than 100 times over the traditional technique using the same equipment [6].

HiBi FLMs present two important drawbacks in practical applications compared to other solutions like FBGs. The first one is the multiplexing capability, being only published a few works up to date in this respect [5-10]. A second important drawback of HiBi FLMs is that in its conventional version (using a single-mode fiber optical coupler), a polarization controller (PC) is required in the loop. This becomes particularly bothersome when multiple sensors are multiplexed, since it is needed one PC per sensing element [5]. Initially proposed in [11] due to its high sensitivity, all-PM fiber loop mirror interferometers can be designed and used even in multiplexing schemes [6, 7]. In this manner, HiBi FLMs can overcome the need of polarization control in the system, which together with its improved multiplexing capability, significantly improve their practical application. Additionally, commercial FBGs interrogators can be adapted by software to interrogate interferometric sensor networks. However, a downside of all-PM FLMs is that the arms of the PM coupler act as sensing elements themselves [6-7, 11], which might not be desirable if specific fibers are used as sensors. The contribution generated by the fiber arms can be obviated; but it increases in a factor of two the number of spatial frequency contributions, limiting the multiplexing capability of the system.

In this work, this effect is suppressed by using a balanced configuration in which the contributions due to the PM optical coupler arms self-compensate between them. Consequently, only the contribution generated by the sensing fiber is present in the system, resulting an equivalent version of the conventional HiBi FLM without the need of polarization control in the loop. This idea can be extended to multiplexing setups so one more sensor can be monitored without increasing the number of frequency contributions. The new all-PM FLM setup has been designed by means of the multi-section theory and it has been validated as temperature and vibration sensor. In this manner, simultaneous monitoring of temperature and vibration has been achieved without crosstalk by using a FFT-based interrogation technique.

*daniel.leandro@unavarra.es; phone +34 94816 9328; fax +34948 16 9720; 


\section{OPERATING PRINCIPLE AND EXPERIMENTAL SETUP}

A fiber loop mirror with a single section of high-birefringence fiber (HiBi-FLM) can be simply formed by connecting the two ports of an optical coupler with a section of HiBi fiber [1]. As a result, the different speeds of the light coupled to the two birefringence axes generate a sinusoidal interference pattern in the coupler which period is defined by the length of HiBi fiber $l$ and its birefringence (also expressed as the beat-length $L_{B}$ ). The sensing principle is based on the detection of small variations of both length and birefringence induced externally in the HiBi fiber (e.g. by temperature changes). Those variations can be understood as phase shift $\Delta \phi$ in the interference pattern. There is also other important factor which is the angle difference of the polarization states entering at both ends of the HiBi section. This angle $\theta$ determines the amplitude of the interference and in the case of a conventional HiBi FLM that uses a single-mode fiber coupler, it is defined by a polarization controller placed inside the cavity.

The proposed interferometer aims to combine the benefits of all-PM interferometers with the simple design and operation of the conventional one-section HiBi FLM. Other all-PM approaches use unbalanced fiber sections so the length difference between the arms of the PM optical coupler defines an additional frequency component in the interference. As a result, if two specific sensing fibers are required in a setup, at least three frequency contributions should be considered in the design, significantly increasing the complexity. However, in this work the contribution given by the communication fibers is avoided by balancing the arms of the PM coupler: both fibers present equal length and are fused with a total angle offset of $90^{\circ}$. Thus, the contribution of the PM fibers is removed, achieving the same system's behavior that the conventional single-section HiBi FLM but with the benefits of an all-PM setup. The theoretical analysis of the structure is performed using equations of the three-section HiBi FLM [7]:

$$
T=\left[C_{1}+C_{2}+C_{3}+C_{4}\right]^{2}
$$

Where:

$$
\begin{aligned}
& C_{1}=\cos \left(\left(\beta_{1}+\beta_{2}+\beta_{3}\right) / 2\right) \sin \left(\theta_{c}\right) \cos \left(\theta_{2}\right) \cos \left(\theta_{3}\right) ; C_{2}=\cos \left(\left(-\beta_{1}+\beta_{2}+\beta_{3}\right) / 2\right) \cos \left(\theta_{c}\right) \sin \left(\theta_{2}\right) \cos \left(\theta_{3}\right) \\
& C_{3}=\cos \left(\left(\beta_{1}+\beta_{2}-\beta_{3}\right) / 2\right) \cos \left(\theta_{c}\right) \cos \left(\theta_{2}\right) \sin \left(\theta_{3}\right) ; C_{3}=-\cos \left(\left(\beta_{1}-\beta_{2}+\beta_{3}\right) / 2\right) \sin \left(\theta_{c}\right) \sin \left(\theta_{2}\right) \sin \left(\theta_{3}\right) \\
& \beta_{n}=\frac{2 \pi \cdot l_{n} \cdot \lambda_{0}}{L_{B n} \cdot \lambda}+\Delta \phi_{n} ;
\end{aligned}
$$

The first condition in the design of the setup is imposed by the PM optical coupler, which angle $\theta_{c}=0^{\circ}$ is fixed [6]. Consequently, since the fibers $l_{1}$ and $l_{3}$ both are Panda SM15-PS-U25A, it is evident using (1) that the fiber lengths must be $l_{1}=l_{3}$ in order to eliminate their contribution. Therefore, $\pm \beta_{1} \mp \beta_{2}=0$. Finally, with the aim of maximizing the interference, the angle offset between fibers is set to $\theta_{2}=\theta_{3}=45^{\circ}$. The result, as stated in (2), is the transfer function $T$ (similarly the reflected response $R$ ) which is equivalent to the response of a conventional one-section HiBi FLM where $\sin \theta=2 \sin ^{2} 45$ [1]. That implies that the interference is only dependent on the sensing fiber length $l_{2}$ and its beat length $L_{B 2}$, being the polarization angle difference at the ports of the coupler a fixed constant. Consequently, no polarization controllers are needed, which significantly simplifies the operation of the system.

$$
T=1-R=\left[2 \sin ^{2} 45 \cos \left(\beta_{2} / 2\right)\right]^{2}
$$
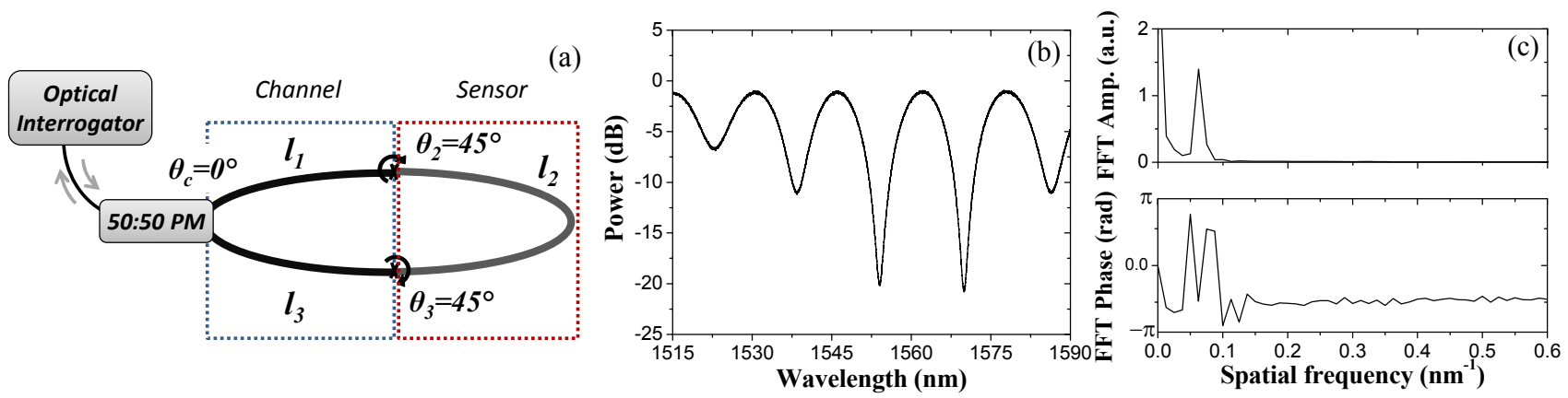

Figure 1. (a) Experimental setup of the proposed HiBi FLM, (b) its optical spectrum and (c) FFT of the optical spectrum. 
The experimental setup consists of a PM optical coupler which arms act as communication fibers [Fig. 1 (a)]. Both fibers are Panda type SM15-PS-U25A with a measured beat length of $3.98 \mathrm{~mm}$ and equal length of $21 \mathrm{~cm}$. Both fiber sections are directly spliced to the sensing fiber with a rotation angle offset of $45^{\circ}$. The sensing fiber is a Panda HB1500 with a beat length of $3.8 \mathrm{~mm}$ and a length of $40 \mathrm{~cm}$. The system is monitored in reflection using a commercial FBG sensor interrogator (Smartec SM125).

\section{EXPERIMENTAL RESULTS}

The measuring technique used in the study is based on the FFT. In this manner, the interrogator is employed in combination with a Matlab software to perform the FFT in real-time $(1 \mathrm{~Hz})$ as in [5]. The results of each optical spectrum provided by the interrogator [Fig. 1 (b)] can be represented in the FFT amplitude and phase spectrum as depicted in Fig. 1 (c). As a consequence, sinusoidal (or a combination thereof) optical signals are characterized in the amplitude FFT spectrum by a peak located at a spatial frequency given by the period of the interference with a magnitude related to the amplitude of the optical signal. Additionally, the phase of the different frequency contributions is displayed in the FFT phase spectrum. It can be seen in Fig. 1 (c) that the spatial frequency contribution of the interferometer is located at $0.0625 \mathrm{~nm}^{-1}$. Thus, the FFT phase at $0.0625 \mathrm{~nm}^{-1}$ will be monitored to obtain the sensing information (temperature in this study). It should be mentioned that the phase value is limited between $\pm \pi$. However, since there are not abrupt temperature changes during the interrogation time (under 1s); the phase data can be easily translated to absolute values by software.
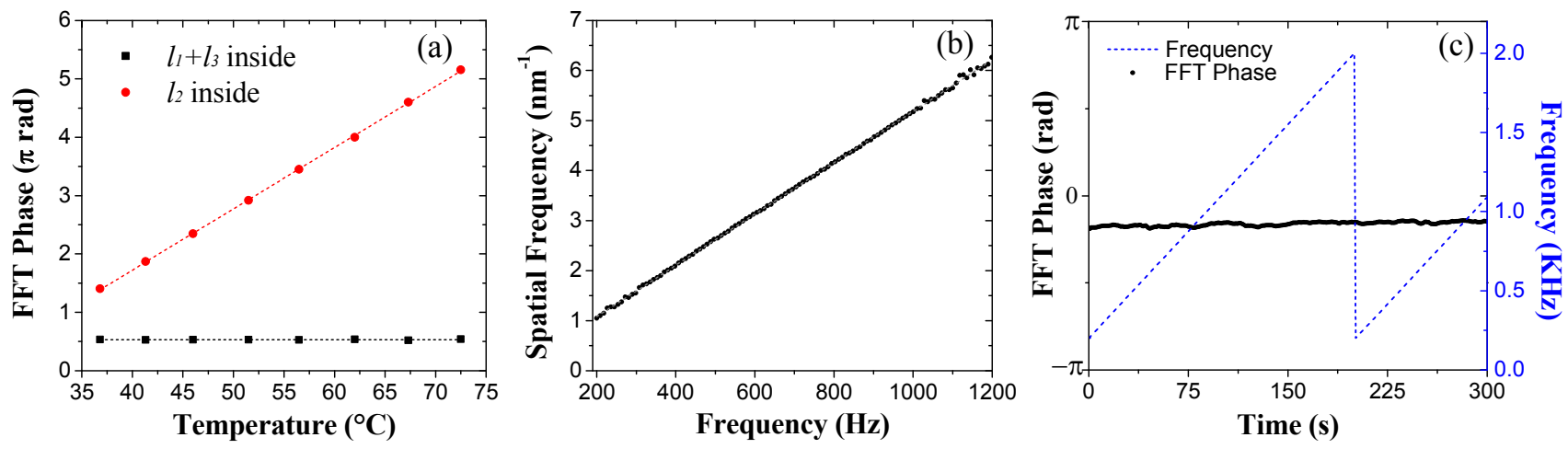

Figure 2. (a) Results of the temperature characterization. (b) Spatial frequency variation with frequency of vibration. (c) FFT phase variation of the sensing fiber as a function of the vibration applied.

Therefore, the temperature of the sensing fiber is retrieved from the value of the FFT phase located at $0.0625 \mathrm{~nm}^{-1}$. First of all, the appropriate operation of the system as temperature sensor was validated by inserting the sensing fiber into a climatic chamber and performing a temperature sweep from 35 to $75^{\circ} \mathrm{C}$. As expected, a linear response can be observed in Fig 2 (a) with a sensitivity of $0.105 \pi \mathrm{rad} /{ }^{\circ} \mathrm{C}$ and a fitting error $\mathrm{R}^{2}=0.9998$. In order to prove the negligible crosstalk between the sensing fiber $\left(l_{2}\right)$ and the communication sections $\left(l_{l}\right.$ and $\left.l_{3}\right)$, a second temperature sweep was performed. In this case, the sensing fiber was kept outside the climatic chamber while the others were subjected to temperature changes from 35 to $75^{\circ} \mathrm{C}$. Fig. 2 (a) (squares) evidences the absence of crosstalk in the measurements, since the FFT phase remained stable during the experiment. Consequently, the setup can be used as temperature sensor without crosstalk.

In the second set of experiments, the system was validated as mechanical vibration sensor. In this case, the interrogation is based on a different concept, by which the modulation induced in the optical signal by the vibration is reflected in the FFT spectrum. In this manner, a new peak is generated in the FFT amplitude spectrum, located at a spatial frequency directly related to the frequency of the vibration. Moreover, the amplitude of the peak is also proportional to the amplitude of the vibration. As a result, vibrations applied to the sensing fiber can be measured by monitoring the spatial frequency and amplitude of the peak generated in the FFT spectrum. The characterization of the device as vibration sensor was carried out employing a mechanical wave driver to apply transversal vibration to the sensing fiber. The pattern of the vibration was a sinusoidal signal which amplitude was reduced by the driver with the increase of the frequency. A frequency sweep was performed between 200 and $1200 \mathrm{~Hz}$, monitoring the spatial frequency of the generated peak as depicted in Fig. 2 (b). It can be seen that the device presents a linear response, showing a sensitivity of $5.13 \mathrm{~nm}^{-1} / \mathrm{kHz}$ and a linear fitting factor $\mathrm{R}^{2}=0.9997$. The vibration frequency resolution is imposed by the spatial frequency resolution 
achieved by the optical interrogator $\left(0.0125 \mathrm{~nm}^{-1}\right)$; consequently obtaining a frequency resolution of $\approx 2.4 \mathrm{~Hz}$. In the same manner, a significantly wider range of frequencies is expected to be measured, being in this experiment the lower limit imposed by the spatial frequency resolution and the higher by the amplitude of the vibration. Further research is being done in this respect. Finally, the absence of crosstalk between vibration and temperature measurements is verified. In this regard, the FFT phase which encodes the temperature information $\left(0.0625 \mathrm{~nm}^{-1}\right)$ is monitored while mechanical vibration is applied to the sensor. As displayed in Fig. 2 (c), there is no evidence of crosstalk since the phase remained constant during the whole frequency sweep. Different vibration amplitudes were also tested with the same result.

\section{CONCLUSION}

In this study, a new all-PM FLM interferometer has been proposed and validated without crosstalk for simultaneous temperature and vibration measurements. The design of the system has been performed by means of the three-section theory (two coupler arms plus the sensing fiber) so that the contributions of the communication fibers are suppressed. To achieve this, the coupler's arms (with equal length) have been fused with a total rotation angle of $45^{\circ}$ with respect to the sensing fiber. In this manner, the setup shows the advantages of all-PM setups (high resolution, accuracy and no need of polarization control) combined with the simplicity of the conventional setup with a well-defined sensing area. It is worth saying that this balanced-arms technique allows extending the number of sensing fibers multiplexed using multi-section FLMs, since there is no contribution generated in the FFT spectrum by the communication fibers (regardless of their length). Moreover, extra fiber sections properly aligned with the communication fibers could be used to create long loops for sensors networks with an interference defined only by the sensing elements. Further work is being done to analyze the properties of the setup and its capability of performing absolute temperature measurements, particularly when compared to the conventional HiBi FLM. The setup has been validated for simultaneous temperature and vibration measurements by means of a FFT-based interrogation technique and a commercial FBG interrogator with a scan frequency of 1 Hz. In this manner, frequencies up to $1.2 \mathrm{kHz}$ have been detected without the need of fast-acquisition interrogators/detectors. In both cases, linear responses have been attained without crosstalk between temperature and vibration.

\section{ACKNOWLEDGEMENTS}

Financial support from the Spanish Comisión Interministerial de Ciencia y Tecnología within projects TEC2013-47264C2-2-R and TEC2016-76021-C2-1-R.

\section{REFERENCES}

[1] Mortimore, D. B., "Fiber Loop Reflectors," Journal of Lightwave Technology 6 (7), 1217-1224 (1988).

[2] Starodumov, A. N. and Zenteno, L. A. and Monzon, D. and De La Rosa, E., "Fiber Sagnac interferometer temperature sensor," Applied Physics Letters, 70, 19-21 (1997).

[3] Hu, Y., Chen, S., "Spatial frequency multiplexing of optical fiber sensor arrays," Optics Letters 20(10), 12071209 (1995).

[4] Barrera, D., Villatoro, J., Finazzi, V. P., Cárdenas-Sevilla, G. A., Minkovich, V. P., Sales, S., Pruneri, V. "Lowloss photonic crystal fiber interferometers for sensor networks," Journal of Lightwave Technology 28(24), 3542-3547 (2010).

[5] Leandro, D., Bravo, M., Ortigosa, A., Lopez-Amo, M., "Real-time FFT analysis for interferometric sensors multiplexing," Journal of Lightwave Technology 33(2), 354-360 (2015).

[6] Leandro, D., Bravo, M., Lopez-Amo, M., "High resolution polarization-independent high-birefringence fiber loop mirror sensor," Optics Express 23(24), 30985-30990 (2015).

[7] Leandro, D., Lopez-Aldaba, A., Bravo, M., Lopez-Amo, M., "Monitoring Multiple Hi-Bi Sensing Fibers in a Single Fiber Loop Mirror," Journal of Lightwave Technology 34(19), 4543-4549 (2016).

[8] Bravo, M., Fernandez-Vallejo, M., Echapare, M., López-Amo, M., Kobelke, J., Schuster, K., "Multiplexing of six micro-displacement suspended-core Sagnac interferometer sensors with a Raman-Erbium fiber laser," Optics Express 21(3), 2971-2977 (2013).

[9] Cardenas-Sevilla, G. A., Finazzi, V., Villatoro, J., Pruneri, V., "Photonic crystal fiber sensor array based on modes overlapping," Optics Express 19(8), 7596-7602 (2011).

[10] Fu, H. Y., et al., "Multiplexing of polarization-maintaining photonic crystal fiber based Sagnac interferometric sensors," Optics Express 17(21), 18501-18512 (2009).

[11] De la Rosa, E., Zenteno, L. A., Starodumov, A. N., Monzon, D., "All-fiber absolute temperature sensor using an unbalanced high-birefringence Sagnac loop,” Optics Letters 22(7), 481-483 (1997). 